\title{
Epigenetics in primary headaches: a new avenue for research
}

\author{
Markus Schürks
}

Received: 3 April 2008/Accepted: 7 April 2008/Published online: 30 April 2008

(C) Springer-Verlag 2008

Sir,

I read with great satisfaction and enthusiasm the review article by Professor Montagna, "The primary headaches: genetics, epigenetics and a behavioural genetic model [1]." He presents a detailed outline of genetic studies in primary headaches, mostly migraine, and at the same time discusses the limitations associated with each of the approaches as mirrored by the inconsistent results. In addition, a comprehensive hypothesis on migraine pathophysiology, incorporating both the Darwinian perspective and recent advances in epigenetics, is presented. This is satisfying in two ways. First, it helps to understand why many of the results are controversial. Most importantly he calls our attention to something we all know: 'migraine' is a collective term for a set of symptoms, as defined by the International Headache Society [2]. This etiological heterogeneity is the main obstacle hampering genetic studies and any small or moderate genetic effect, that may be important for a subgroup of migraines of the same origin (same underlying syndrome or disease), will inevitably be diluted when we look at all migraines. Furthermore, environmental aspects (nutrients, living conditions, etc., with the potential to exert epigenetic changes) have so far been disregarded in genetic studies. Second, this novel hypothesis on migraine pathophysiology incorporates existing knowledge into a new, more complex framework, rather

Reply to this letter can be found at doi:10.1007/s10194-008-040-z.

M. Schürks ( $\square)$

Division of Preventive Medicine,

Brigham and Women's Hospital,

900 Commonwealth Avenue East,

3rd fl, Boston, MA 02215-1204, USA

e-mail: mschuerks@ rics.bwh.harvard.edu than refuting prior studies. This encourages continuing to search for the origin of primary headaches.

Epigenetics refers to the heritability of traits not attributable to a change in DNA sequence. Two common mechanisms are DNA methylation and post-translational histone modification [3]. Although a relatively young research branch, tremendous progress has been made in elucidating how, e.g., DNA methylation regulates gene expression and how that impacts disease susceptibility, in particular cancerogenesis [3]. In addition, pharmacological approaches to modify DNA methylation and, thus, gene expression have come into focus recently [4]. In extension to Professor Montagna's 'behavioural genetic model' of migraine and other primary headache disorders, I would also like to suggest the idea of a epigenetic cause for medication response. In migraine abortive and preventive drugs are effective in only about half of patients [5] and in cluster headache subcutaneous sumatriptan is effective in $80 \%$ of patients at best $[6,7]$. These phenomena may be explained by functional gene variants [8]. However, response to analgesics and triptans can also change over time and depending on the frequency of usage (medication overuse headache impairing effectiveness of abortive medication). This cannot be explained by simple alterations in the genetic code, but suggests epigenetic changes at target molecules. Such a 'pharmaco(epi-)genetic' model would acknowledge both DNA sequence variation and methylation, etc., as determinants of drug treatment response.

This article [1] fuels headache research enthusiasm. Admittedly, the proportion epigenetics plays with regard to disease causation and drug response is unknown and epigenetics will unlikely provide answers to all open questions in headache research. However, I consider this 'behavioural genetic model' a very valuable framework 
allowing for reconsideration of the pathophysiology and pharmacogenetics of primary headaches.

\section{Conflict of interest None.}

\section{References}

1. Montagna P (2008) The primary headaches: genetics, epigenetics and a behavioural genetic model. J Headache Pain 9:57-69. DOI 10.1007/s10194-008-0026-x

2. International Headache Society (2004) The international classification of headache disorders, 2nd edn. Cephalalgia 24(Suppl 1):9160
3. Jirtle RL, Skinner MK (2007) Environmental epigenomics and disease susceptibility. Nat Rev Genet 8:253-262

4. Peedicayil J (2006) Epigenetic therapy-a new development in pharmacology. Indian J Med Res 123:17-24

5. Goadsby PJ, Lipton RB, Ferrari MD (2002) Migraine-current understanding and treatment. N Engl J Med 346:257-270

6. The Sumatriptan Cluster Headache Study Group (1991) Treatment of acute cluster headache with sumatriptan. N Engl J Med 325:322-326

7. Schürks M, Kurth T, de Jesus J, Jonjic M, Rosskopf D, Diener HC (2006) Cluster headache: clinical presentation, lifestyle features, and medical treatment. Headache 46:1246-1254

8. Schürks M, Kurth T, Stude $P$ et al (2007) G protein beta3 polymorphism and triptan response in cluster headache. Clin Pharmacol Ther 82:396-401 